\title{
Introduction: Intracranial Aneurysm Surgery
}

\author{
Daniel L. Barrow, M.D. ${ }^{1}$ and Ralph Dacey, M.D. ${ }^{2}$ \\ 1Department of Neurosurgery, Emory University School of Medicine, Atlanta, Georgia; and 2Department of Neurosurgery, \\ Washington University School of Medicine, St. Louis, Missouri
}

This Neurosurgical Focus video supplement includes narrated videos of a variety of surgical approaches to intracranial aneurysms. Contributions include common aneurysms requiring straightforward approaches as well as very complex lesions that challenge the skills of neurovascular surgeons. These video presentations illustrate the fact that microsurgery remains a time-honored, versatile and durable therapeutic option for the management of many intracranial aneurysms. Despite the dramatic advances in endovascular therapy, microsurgery is not a static endeavor and remains the optimal treatment for a substantial number of aneurysms. Many of the tools that have contributed to the advancement of microsurgery are illustrated in the case presentations. These include the use of skull base techniques, minimally invasive approaches, bypass surgery, thrombectomy, the use of temporary and modern aneurysm clips, brain protection and other technical advances.
Some of the included videos are abbreviated to show particular surgical nuances while other videos are more comprehensive to demonstrate imaging, operative set up and positioning, exposure as well as surgical strategies. Any redundancy is to provide the perspective of different approaches to a problem.

It has been a privilege to serve as guest editors for this video supplement. We would like to express our appreciation to Margie Shreve, the Focus Editorial Assistant for all of her help. We owe a great thanks to Sander Connolly, Loch McDonald and Brian Hoh for assisting with the review of many of the submitted videos. We would also like to thank all of the authors for sharing their insight, knowledge and skills that helped make up this video supplement. Most of all, and on behalf of all the contributors, we thank our patients who have placed their trust in us for their care. 